\title{
EXTENDED WHOLE MESH DEFORMATION MODEL: FULL 3D PROCESSING
}

\author{
Przemyslaw Lenkiewicz ${ }^{1}$, Manuela Pereira ${ }^{1}$, Mário M. Freire ${ }^{1}$, José Fernandes ${ }^{2}$ \\ ${ }^{1}$ IT-Networks \& Multimedia Group, Department of Computer Science, University of Beira Interior, \\ Rua Marquês d'Ávila e Bolama, 6201-001, Covilhã, Portugal \\ ${ }^{2}$ Microsoft Portugal, Av. Prof. Doutor Aníbal Cavaco Silva, 2744-010 Porto Salvo, Portugal
}

\begin{abstract}
Processing medical data has always been an interesting field that has shown the need for effective image segmentation methods. Modern medical image segmentation solutions are focused on 3D image volumes, which originate at advanced acquisition devices. Operating on such data in a $3 \mathrm{D}$ environment is essential in order to take the full advantage of the available information. In this paper we present an extended version of our 3D image segmentation and reconstruction model that belongs to the family of Deformable Models and is capable of processing large image volumes in competitive times and in fully 3D environment, offering a big level of automation of the process and a high precision of results. It is also capable of handling topology changes and offers a very good scalability on multi-processing unit architectures. We present a description of the model and show its capabilities in the field of medical image processing.
\end{abstract}

\section{INTRODUCTION}

Segmentation of medical image data has proven to be an important component of the modern Computer Aided Diagnostic solutions. After such treatment the medical images present a set of data that is far more readable for medical practitioner and more suitable for further algorithmic processing. In the last years the research efforts in that field have been surrounded on 3D medical imaging, produced by the modern medical scanners. These acquisition devices are capable of delivering 3D volumes composed of a series of 2D slice images of high spatial resolution and with low distances between consecutive slices. Using existing 2D image segmentation algorithms on this $3 \mathrm{D}$ data was usually limited to segmenting each of the component slices separately and then combining the result to create a full 3D representation. This has proven to be not effective and thus a group of algorithms designed specifically for the $3 \mathrm{D}$ images has been designed $[1,2]$. In contrast to the $2 \mathrm{D}$ segmentation solutions, they allowed to incorporate all information present in the 3D input volume at one instance of the segmentation task and therefore to take better advantage of the available data and produce a more accurate result. They have also allowed to include the available medical knowledge about the dimension and shape of different anatomical parts in the segmentation process.

In $[3,4]$ we have presented our 3D image segmentation and reconstruction solution, called the Whole Mesh Deformation Model (WMD). It has been designed using the ideas of the Deformable Models (DM) [5], [6] algorithm family, which has proven to be very successful and effective in numerous fields of application. Like in most solutions from the DM family, the segmentation process in WMD is based on optimization of the energy function, which is calculated taking the current shape of the model as the input. The WMD model has proven to be a very promising solution as it is able to deliver precise results in very competitive execution times and is highly suitable for parallelization. It has however lacked an important feature, which was the ability to deform the mesh in full 3D environment.

\section{3D VERSUS SEMI-3D PROCESSING}

In the first version of the WMD model the distribution of the mesh upon initialization is defined by the following set of parameters:

The distances between the nodes in $\mathrm{X}$ and $\mathrm{Y}$ directions are defined by the user. Smaller distances result with bigger density of the mesh and larger precision of the final result. These values could have been chosen completely freely.

The distance between layers of the mesh in $\mathrm{Z}$ direction is defined by the number of slice images in the input volume. Each input slice corresponds to exactly one layer of nodes in the mesh and the distance between them (in voxels) has to be passed as a parameter by the user.

During the segmentation process the nodes are allowed to move in $\mathrm{X}$ and $\mathrm{Y}$ directions in order to explore the information available in the input images. Movement in the $\mathrm{Z}$ direction was not allowed, because the progression by even one voxel in that dimension would mean moving out of the input image and therefore loosing the access to any image information, which plays a vital part in the entire segmentation process.

Although this approach has shown to be a very good tradeoff between precision and segmentation times, there are some 


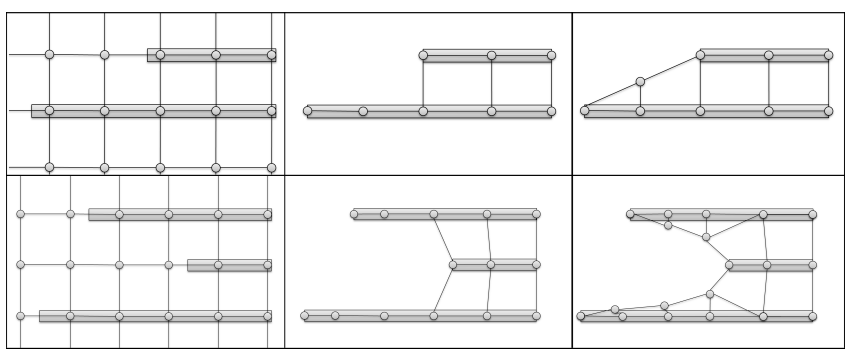

Fig. 1. Two examples of situations in which the 2D processing of the nodes would perform not satisfactory. The top-left and bottom-left images present the initial distribution of the mesh in both examples. Images in the middle and on the right present respectively the results obtained with 2D and 3D node movement approaches.

situations for which it might prove not good enough. In some situations the difference between two consecutive slices can be very significant and nodes that are fixed to a specific $\mathrm{Z}$ plane might not be able to properly describe the objects of interests. Two such examples are shown on Fig. 1.

The proper functionality to handle these cases could be obtained with the implementation of node movement in the $\mathrm{Z}$ direction. The initialization of the $\mathrm{Z}$ planes with the $1: 1$ correspondence to the number of input slices should still be maintained as the most optimal approach. The justification for this is the following: the WMD model is constructed in a way to decrease as much as possible the distance which the nodes need to travel during the segmentation task. Our experiments have proven that the majority of the nodes of the mesh do not propagate away from their initial positions farther than half of the distance between the nodes in the initial distribution of the mesh. This will be also true if the nodes have the ability to move in the $\mathrm{Z}$ direction, their movement would not exceed the half of the distance between consecutive $\mathrm{Z}$ planes. Therefore, implementation of more complex mechanism that would allow initialization of the mesh independently from the number of input image slices, would not allow any new functionality or increased precision of the result and it could result with higher computational cost of the method.

\section{DEFINITION OF FULL 3D NODE MOVEMENT}

\subsection{Movement of the nodes}

In the original formulation of the WMD the nodes have been allowed to move only in $\mathrm{X}$ and $\mathrm{Y}$ directions. This step has been realized using the Greedy algorithm optimization approach [7]. During the procedure the following is performed for each node $N$ of the mesh: if the coordinates

$$
N_{t}=\left(x_{n}, y_{n}, z_{n}\right)
$$

describe the position of the node $N$ at the time $t$, then for time $t+1$ the coordinates of $N$ would be described with

$$
N_{t+1}=\left(x_{n+k}, y_{n+l}, z_{n}\right)
$$

where $k, l \in\{-1,0,1\}$ and correspond to the location with the lowest possible value of the energy function. In order to allow the nodes to move also in the $\mathrm{Z}$ direction we need to enrich (2) with the following:

$$
N_{t+1}=\left(x_{n+k}, y_{n+l}, z_{n+m}\right)
$$

where also $m \in\{-1,0,1\}$ and the combination of $k, l, m$ corresponds to the lowest possible value of the energy function. As we can see, the extended are of movement will be constructed from 27 instead of 9 points and the node will be moved in its nearest neighborhood in $X, Y$ and $Z$ planes. Similarly, the position with the lowest energy will be chosen as the new position. Those steps will be repeated for each node of the mesh until the following rule is satisfied:

$$
\sum \operatorname{dist}_{m}\left(N_{t}, N_{t+1}\right)<\mu
$$

where $\mu$ is a small value near zero. Equation (4) verifies the number of nodes that have changed their position in the last algorithm iteration. Whenever this number is decreased to zero (or very near to zero) the mesh is assumed to be in its stable position and the segmentation is finished.

\subsection{Formulation of the Energy Function}

The segmentation process in the fully 3D WMD model will be carrier out in the same manner as in the original WMD formulation. The energy function would be defined to represent the internal features of the model and the data in the input images. This function would be calculated taking the current shape of the mesh as the input. The state of the mesh corresponding to the lowest value of the energy function would be considered as the solution of the segmentation task.

Since the optimization process has been significantly modified, the energy function and its numerical parameters need to be properly updated, to enforce the correct behavior of the mesh. As it can be seen on Fig. 1, in certain situations we would like the nodes of the mesh to progress away from their original $\mathrm{Z}$ plane. This can be generally described as situations when two of more consecutive slices of the input volume contain objects with their edges distributed farther from each other than the distance between the nodes in the initial distribution of the mesh. This can also be seen on Fig. 1 right. Some nodes of the mesh move in the $\mathrm{Z}$ direction in order to construct a smoother surface between the planes of the input volume and thus to create a more realistic segmentation result. In order to enforce such behavior on the 
mesh we can include the following mechanisms in the mesh deformation scheme:

- The links of the mesh should show a subtle shrinking trend. This will attract some of the nodes to move away from their $\mathrm{Z}$ plane in situations like the ones shown on Fig. 1, namely when their neighboring nodes are located next to the edges of the objects of interests.

- The flexibility of the links of the mesh should be slightly increased comparing to the previous version of the WMD model. This means that they should be allowed to extend to larger lengths before they will be broken by the topology changing mechanism [3]. Fig. 1 shows that keeping a larger number of links from breaking will help to attract the necessary nodes to assume their positions between two $\mathrm{Z}$ planes, where they would normally stay if links connecting them with neighbors would have been broken.

The acquisition of the above mentioned features into the WMD model might not guarantee that the mesh will behave exactly as shown on Fig. 1, namely create a ideally regular shape, but the desired and acceptable feature is that some of the nodes would position themselves between the original $\mathrm{Z}$ planes and create a cloud of points, which would connect the edges of objects on neighboring image slices and create a smooth transition between them.

\subsection{Calculation of External Energy Outside of the Input Images}

Calculation of the external energy function in the first formulation of the WMD model was performed for each individual node of the mesh using the greyscale values of three images at the $\mathrm{X}$ and $\mathrm{Y}$ coordinates of that given node. The images composing the external energy have been the following: the input image intensity values, Gradient Vector Flow (GVF) [8] and Canny Edge Detector [9] (the last two images have been created from the input data in the beginning of the segmentation task). In situation where we would like to allow the nodes to propagate away from their original $\mathrm{Z}$ plane, we need to provide a new way of calculating the external energy, as the nodes would no longer be assuming positions which the data in the input images describe. In order to provide that functionality we have defined the new external energy as follows:

$$
\begin{aligned}
E_{e x t}\left(\nu_{x, y, z}\right)= & \gamma \sum_{n=1}^{k}\left(1-I\left(\nu_{n}\right)\right)+\delta \sum_{n=1}^{k}\left(1-G\left(\nu_{n}\right)\right)+ \\
& +\epsilon \sum_{n=1}^{k} E\left(\nu_{n}\right)
\end{aligned}
$$

where $\nu_{x, y, z}$ is a mesh node $\nu$ with $x, y, z$ coordinates, $k$ is the total number of nodes in the mesh, $I(\nu), G(\nu)$ and $E(\nu)$ are respectively the intensity value, GVF value and edge detector value corresponding to that node. They are calculated in the following way:

$$
I\left(\nu_{n}\right)=I\left(\nu_{x, y, z_{1}}\right) \times s 1+I\left(\nu_{x, y, z_{2}}\right) \times s 2
$$

where $I\left(\nu_{x, y, z_{1}}\right)$ and $I\left(\nu_{x, y, z_{2}}\right)$ represent the intensity values from the input images with coordinates $x, y, z_{1}$ and $x, y, z_{2}$ respectively, $z_{1}$ and $z_{2}$ represent the $Z$ coordinates of the two planes between which the given node is located, $s_{1}$ and $s_{2}$ represent the weights of both intensity values and are calculated taking the following value into consideration:

$$
s_{i}=\frac{1}{\operatorname{dist}_{s}\left(N_{t}(x, y, z), N_{t}\left(x, y, z_{i}\right)\right)}
$$

As we can, equations 6 and 7 compute the intensity value of node $\nu_{x, y, z}$ using the values corresponding to nodes $\nu_{x, y, z_{1}}$ and $\nu_{x, y, z_{2}}$, which are weighted accordingly to the distance of the current node in $\mathrm{Z}$ plane from the two planes surrounding it. Using this approach we are able to project to a certain extend the information that would be present between the available image slices. It offers a good level of realism and approximation of the real data. For the remaining two types of images used in our energy calculation the situation would be similar: for the values of intensity calculated between two image slices we would see a graduate progression of the values from one slice to another. In the case of edge detector images the situation is slightly different, because those images are interpreted in the following way: any value other than 0 represents an edge and all the non-edge area is represented by 0 . However, our mechanism would still perform desirably, as it would mark all the area between two slices as a non-edge area and thus it would allow the two remaining external energy components to model the behavior of the node in that areas. The general assumptions of this method make it very suitable for our goals. The precision of the final result of the segmentation can be fine-tuned by the numerical parameters of the energy function.

\section{RESULTS}

We have implemented the segmentation method based on the extended model described in Section 3. The experiments included segmentation of three medical image volumes: a 256x256x11 CT brain scan, a 256×256x10 CT brain scan and a 472x512x16 MRI knee scan. Fig. 2 presents the execution times for each segmentation task. As it can be seen, if we choose only $2 \mathrm{D}$ movement of the nodes of the mesh, the results are delivered in shorter times. This has been expected as each node is positioned in 27 instead of 9 locations in each iteration of the algorithm. The execution times are around $52 \%$ longer in each of the experiments. 


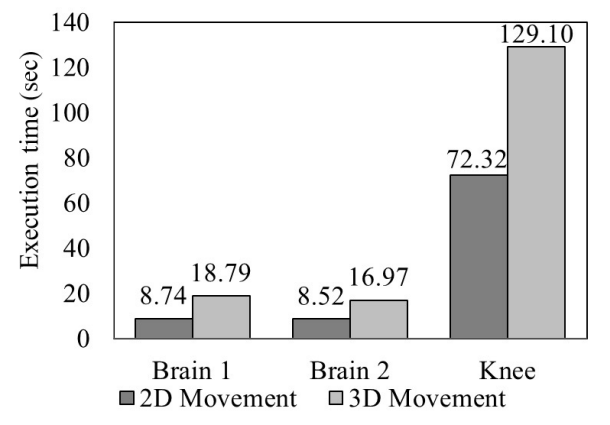

Fig. 2. Execution times (in seconds) for 3 segmentation experiments presented in this section.

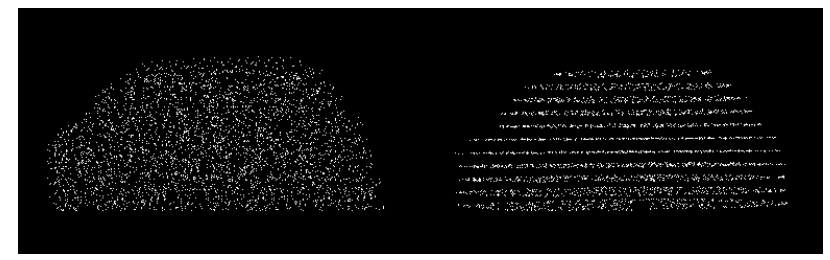

Fig. 3. Results of the CT brain scan segmentation experiment. Left: full 3D movement of nodes, right: only $2 \mathrm{D}$ movement of nodes

Fig. 3 presents an exemplary result obtained from the CT brain scan using the two version of the algorithm. The 3D movement capable version is presented on the left and the 2D movement only version is shown on the right. The images present the final result projected from the Y plane, which means that the $\mathrm{X}$ and $\mathrm{Z}$ planes appear visible. Only the nodes of the mesh are presented, the links have been hidden. As it can be seen, the 3D movement has produced a volume with nodes that are distributed in all the planes. On the other hand, the 2D movement version has produced a volume with nodes restricted to their original $\mathrm{Z}$ coordinates. As we can expect, this sort of result would not describe properly the real-world objects. As it can be seen from Fig. 3, using the 3D movement of the nodes we are able to reconstruct the segmented object faithfully and produce a desirable result.

\section{CONCLUSIONS}

In this paper we have presented an extended version of our previous work, the Whole Mesh Deformation Model for 3D image segmentation and reconstruction. The new version has provided a very important feature, namely to process the images in fully $3 \mathrm{D}$ environment by allowing the movement of the control points in all 3 dimensions. Using the new approach we have achieved the possibility to produce a more realistic and smooth reconstruction of the objects of interest. The drawback of the extended model is the increased computational cost. However the overhead is not large and in cases where full 3D movement is not justified, it would still be possible to restrict the nodes of the WMD model to pure 2D movement.

\section{REFERENCES}

[1] T. Mcinerney and D. Terzopoulos, "Topology adaptive deformable surfaces for medical image volume segmentation," IEEE Transactions on Medical Imaging, vol. 18, pp. 840-850, 1999.

[2] M. I. Malladi, R. Kimmel, D. Adalsteinsson, G. Sapiro, and J. A. Sethian, "A geometric approach to segmentation and analysis of," in In IEEE Workshop on Mathematical Methods in Biomedical Image Analysis. IEEE Computer Society Press, 1996, pp. 244-252.

[3] P. Lenkiewicz, M. Pereira, M. Freire, and J. Fernandes, "The dynamic topology changes model for unsupervised image segmentation," in Multimedia Signal Processing, 2009. MMSP '09. IEEE International Workshop on, oct. 2009, pp. $1-5$.

[4] P. Lenkiewicz, M. Pereira, J. Fernandes, and M. Freire, "The whole mesh deformation model for $2 \mathrm{~d}$ and $3 \mathrm{~d}$ image segmentation," in Proceedings 16th IEEE International Conference on Image Processing (ICIP), 2009, pp. 40454048.

[5] M. Kass, A. Witkin, and D. Terzopoulos, "Snakes: Active contour models," International Journal of Computer Vision, vol. 1, no. 4, pp. 321-331, January 1988.

[6] V. Caselles, R. Kimmel, and G. Sapiro, "Geodesic active contours," in Proc. 5th International Conference on Computer Vision, Massachusetts, June 1995.

[7] D. J. Williams and M. Shah, "A fast algorithm for active contours and curvature estimation," CVGIP: Image Underst., vol. 55, no. 1, pp. 14-26, 1992.

[8] C. Xu and J. Prince, "Gradient vector flow: A new external force for snakes," in Proc. IEEE Conference on Computer Vision and Pattern Recognition, 1997, pp. 66-71.

[9] J. Canny, "A computational approach to edge detection," IEEE Transaction on Pattern Analysis and Machine Intelligence, vol. 8, no. 6, pp. 679-698, November 1986. 